\title{
Permanence and uniform asymptotical stability of a ratio-dependent Leslie system with feedback controls on time scales
}

\author{
Zhouhong $\mathrm{Li}^{\mathrm{a}}$, Jianwen Zhou ${ }^{\mathrm{b}}$, Tianwei Zhang ${ }^{\mathrm{c}, *}$ \\ a Department of Mathematics, Yuxi Normal University, Yuxi 653100 China \\ b Department of Mathematics, Yunnan University, Kuming 650091 China \\ c Institute de Mathématiques, Kunming University of Science and Technology, Kunming 650500 China
}

*Corresponding author, e-mail: zhang@kust.edu.cn

Received 3 Jan 2020

Accepted 5 Jul 2020

\begin{abstract}
Using time-scale calculus and construction of a suitable Lyapunov functional, the permanence and uniform asymptotical stability of a ratio-dependent Leslie model with feedback control on time scales are studied. The results of this paper extend some recent research results. An illustrative example with numerical simulations is employed to visually manifest the theoretical findings.
\end{abstract}

KEYWORDS: permanence, uniform asymptotical stability, Leslie system, feedback control, time scale

MSC2010: 34D20 92D25

\section{INTRODUCTION}

Predator-prey is one of the important interactions among species commonly observed in social animals and human society. The dynamical relationship between predators and their preys has long been and will continue to be one of the dominant themes in both ecology and mathematical ecology due to its universal existence and importance. Leslie [1] proposed a predator-prey model to describe the "carrying capacity" of the predator's environment that is proportional to the number of preys. Leslie emphasized that the rates of increase of both preys and predators are limited, which is not the same as those in the Lotka-Volterra model. In case the number of preys is large or the number of predators is small, the rates are able to reach their upper limits. In terms of continuous time, these discussions deduce Leslie predator-prey system as follows:

$$
\left\{\begin{array}{l}
\dot{x}_{1}=x_{1}\left[b-a x_{1}\right]-p\left(x_{1}, x_{2}\right) x_{2}, \\
\dot{x}_{2}=x_{2}\left[g-f \frac{x_{2}}{x_{1}}\right]
\end{array}\right.
$$

where $x_{1}$ and $x_{2}$ stand for the population (the density) of the preys and of the predators, respectively, $p$ is the so-called predator functional response to predator and prey. In the last decades, the dynamical behaviors for the continuous-time Leslie predator-prey systems such as Hopf bifurcation $[2,3]$, permanence $[4]$, periodic solution $[5,6]$, almost periodic solution $[7,8]$, and stability [4], etc., have been widely investigated.

Recently, more and more obvious evidences of biology and physiology showed that in many conditions, especially when the predators have to search for food (consequently, have to share or compete for food), a more realistic and general predatorprey system should rely on the theory of ratiodependence, this theory is confirmed by lots of experimental results. In the last decades, much work has been done on the ecosystem with feedback controls $[9,10]$. In particular, Wang et al [11] considered a ratio-dependent Leslie predator-prey model with feedback controls as follows:

$$
\left\{\begin{aligned}
\dot{x}_{1}(t)= & x_{1}(t)\left[b(t)-a(t) x_{1}(t)\right. \\
& \left.-\frac{c(t) x_{1}(t) x_{2}(t)}{h^{2}(t) x_{2}^{2}(t)+x_{1}^{2}(t)}-d(t) u_{1}(t)\right], \\
\dot{x}_{2}(t)= & x_{2}(t)\left[g(t)-f(t) \frac{x_{2}(t)}{x_{1}(t)}-p(t) u_{2}(t)\right], \\
\dot{u}_{i}(t)= & \alpha_{i}(t)-\beta_{i}(t) u_{i}(t)+\gamma_{i}(t) x_{i}(t),
\end{aligned}\right.
$$

where $u_{1}(t)$ and $u_{2}(t)$ are control variables. Under the assumption that the coefficients of the above system are all $T$-periodic functions, they obtained the existence of a unique globally attractive positive $T$-periodic solution of the above system.

Many literatures have discussed that the discrete-time systems described by difference equations are more realistic and more appropriate than the continuous-time systems in case 
there exists non-overlapping generations in the populations. Furthermore, discrete-time systems can also provide efficient computational process of continuous-time systems for numerical simulations. As a result, various dynamical behaviors of discrete-time Leslie-Gower predator-prey systems, such as Bifurcations [12,13], chaos control [12], chaos [13], permanence $[14,15]$, almost periodic solutions $[14,15]$, and stability [14], have become research highlights for many scholars

In [15], the following discrete ratio-dependent Leslie model was discussed:

$$
\left\{\begin{aligned}
x_{1}(n+1)= & x_{1}(n) \exp \left\{b(n)-a(n) x_{1}(n)\right. \\
& \left.-\frac{c(n) x_{1}(n) x_{2}(n)}{h^{2}(n) x_{2}^{2}(n)+x_{1}^{2}(n)}-d(n) u_{1}(n)\right\}, \\
x_{2}(n+1)= & x_{2}(n) \exp \left\{g(n)-f(n) \frac{x_{2}(n)}{x_{1}(n)}-p(n) u_{2}(n)\right\}, \\
\Delta u_{i}(n)= & \alpha_{i}(n)-\beta_{i}(n) u_{i}(n)+\gamma_{i}(n) x_{i}(n),
\end{aligned}\right.
$$

where $\Delta u_{i}(n)=u_{i}(n+1)-u_{i}(n) ; \alpha_{i}, \beta_{i}, \gamma_{i}, d, p$, $h, a, b, c, f, g$ are bounded sequences defined on $\mathbb{Z}^{+}, i=1,2 ; \mathbb{Z}^{+}$is the set of nonnegative integers. The author obtained the permanence and the existence of a unique globally attractive positive almost periodic solution for the above discrete-time system.

Time-scale calculus was proposed in 1990, which unify the continuous-time analysis and the discrete-time analysis [16]. Later on, the theory of time scales was mainly developed by Bohner and Peterson $[17,18]$. Recently, the theory of time scales has been applied in neural networks [19], ecological systems [20], as well as a variety of control systems [21]. With the mind of time scale scheme, the above continuous-time system (1) and discretetime system (2) can be unified and integrated. It is therefore meaningful to study the dynamic systems on time scales that can unify differential and difference systems. Motivated by the results mentioned above, in this paper, we are concerned with the following ratio-dependent Leslie model with feedback controls on time scales:

$$
\left\{\begin{aligned}
N_{1}^{\Delta}(t)= & b(t)-a(t) \exp \left\{N_{1}(t)\right\}-d(t) u_{1}(t) \\
& -\frac{c(t) \exp \left\{N_{1}(t)+N_{2}(t)\right\}}{h^{2}(t) \exp \left\{2 N_{2}(t)\right\}+\exp \left\{2 N_{1}(t)\right\},} \\
N_{2}^{\Delta}(t)= & g(t)-f(t) \exp \left\{N_{2}(t)-N_{1}(t)\right\}-p(t) u_{2}(t), \\
u_{i}^{\Delta}(t)= & \alpha_{i}(t)-\beta_{i}(t) u_{i}(t)+\gamma_{i}(t) \exp \left\{N_{i}(t)\right\},
\end{aligned}\right.
$$

$t \in \mathbb{T}, i=1,2$, where $\mathbb{T}$ is a time scale, which will be defined in Definition $1 ; b$ and $g$ represent population growth rates of prey and predator, respectively; $a$ stands for the prey death rate; $f$ is the maximum value which represents per capita reduction rate of prey; $c$ denotes the maximum value which per capita reduction rate of prey can attain; $h$ is the interference parameter; $b(t), a(t)$, $c(t), d(t), h(t), g(t), f(t), p(t), \alpha_{i}(t), \beta_{i}(t)$ and $\gamma_{i}(t)$ are nonnegative functions, in which $i=1,2$. If $\mathbb{T}=\mathbb{R}$, then (3) is reduced to (1) and if $\mathbb{T}=\mathbb{Z}$, then system (3) is reduced to (2), where $\mathbb{R}$ and $\mathbb{Z}$ are the set of real numbers and the set of integers, respectively.

\section{PRELIMINARIES}

Definition $1[17,18]$ Let $\mathbb{T}$ be a nonempty closed subset (time scales) of $\mathbb{R}$. For any subset $\mathbb{I}$ of $\mathbb{R}$, we denote $I_{\mathbb{T}}=I \cap \mathbb{T}$. The forward and backward jump operators $\sigma, \rho: \mathbb{T} \rightarrow \mathbb{T}$ and the graininess $\mu: \mathbb{T} \rightarrow$ $\mathbb{R}_{+}$are defined, respectively, by

$$
\begin{aligned}
& \sigma(t)=\inf \{s \in \mathbb{T}: s>t\}, \\
& \rho(t)=\sup \{s \in \mathbb{T}: s<t\}, \\
& \mu(t)=\sigma(t)-t .
\end{aligned}
$$

For $y: \mathbb{T} \rightarrow \mathbb{R}$ and $t \in \mathbb{T}^{k}$, we define the delta derivative of $y(t), y^{\Delta}(t)$, to be the number (if it exists) with the property that, for any $\epsilon>0$, there exists a neighborhood $U$ of $t$ such that

$$
\left|[y(\sigma(t))-y(s)]-y^{\Delta[\sigma(t)-s]}\right|<\epsilon|\sigma(t)-s|
$$

for all $s \in U$. Let $y$ be right-dense continuous. If $Y^{\Delta}(t)=y(t)$, then we define delta integral by

$$
\int_{a}^{t} y(s) \Delta(s)=Y(t)-Y(a) .
$$

A function $p: \mathbb{T} \rightarrow \mathbb{R}$ is called regressive provided $1+\mu(t) p(t) \neq 0$ for all $t \in \mathbb{T}^{k}$. The set of all regressive and rd-continuous functions $p: \mathbb{T} \rightarrow \mathbb{R}$ will be denoted by $\mathfrak{R}=\mathfrak{R}(\mathbb{T})=\mathfrak{R}(\mathbb{T}, \mathbb{R})$. If $\mu(t) p(t)>0$ for all $t \in \mathbb{T}$, then $p$ is a positively regressive function from $\mathbb{T}$ to $\mathbb{R}$. If $r$ is a regressive function, then the generalized exponential function $e_{r}$ is defined by

$$
e_{t}(t, s)=\exp \left\{\int_{s}^{t} \xi_{\mu(\tau)}(r(\tau)) \Delta \tau\right\}, s, t \in \mathbb{T},
$$

with the cylinder transformation

$$
\xi_{h}(z)= \begin{cases}\frac{\log (1+h z)}{h}, & h \neq 0, \\ z, & h=0 .\end{cases}
$$

Definition 2 [22] For each $t \in \mathbb{T}$, let $\mathbb{N}$ be a neighbourhood of $t$. Then we define the generalized derivation (of Dini derivative), $D+u \Delta(t)$, to mean 
that, given $\epsilon>0$, there exists a right neighbourhood $N(\epsilon>0) \subset N$ of $t$ such that

$$
\frac{u(\sigma(t))-u(t)}{\sigma(t)-s}<D^{+} u^{\Delta}(t)+\epsilon
$$

for each $s \in N(\epsilon>0), s>t$. In case $t$ is rightscattered and $u(t)$ is continuous at $t$, this reduces to

$$
D^{+} u^{\Delta}(t)=\frac{u(\sigma(t))-u(t)}{\sigma(t)-s} .
$$

Lemma 1 [17] The following facts hold:

(1) $\left(v_{1} f+v_{2} g\right)^{\Delta}=v_{1} f^{\Delta}+v_{2} g^{\Delta}$, for any constants $v_{1}$ and $v_{2}$;

(2) $(f g)^{\Delta}=(t)=f^{\Delta}(t) g(t)+f(\sigma(t)) g^{\Delta}(t)=$ $f(t) g^{\Delta}(t)+f^{\Delta}(t) g(\sigma(t))$

(3) if $f^{\Delta} \geqslant 0$, then $f$ is nondecreasing.

Lemma 2 [23] Let $-a \in \mathfrak{R}$. The following facts are valid:

(1) If $N^{\Delta}(t) \leqslant b-a N^{\alpha}(t)$, then for $t>t_{0}$,

$N(t) \leqslant N\left(t_{0}\right) e_{(-a)}\left(t, t_{0}\right)+\left(\frac{b}{a}\right)^{\alpha}\left(1-e_{(-a)}\left(t, t_{0}\right)\right)$.

In particular, if $a, b>0$, we have $\lim \sup _{t \rightarrow \infty} N(t) \leqslant\left(\frac{b}{a}\right)^{\alpha}$, where $\alpha$ is a positive constant.

(2) If $N^{\Delta}(t) \geqslant b-a N^{\alpha}(t)$, then for $t>t_{0}$,

$N(t) \geqslant N\left(t_{0}\right) e_{(-a)}\left(t, t_{0}\right)+\left(\frac{b}{a}\right)^{\alpha}\left(1-e_{(-a)}\left(t, t_{0}\right)\right)$.

In particular, if $a, b>0$, we have $\liminf _{t \rightarrow \infty} N(t) \geqslant\left(\frac{b}{a}\right)^{\alpha}$, where $\alpha$ is a positive constant.

\section{PERMANENCE}

Definition 3 System (3) is said to be permanent if for any solution $\left(N_{1}(t), N_{2}(t), u_{1}(t), u_{2}(t)\right)^{\mathrm{T}}$ of system (3), there exist some constants $m_{i}, q_{i}, M_{i}$ and $Q_{i}(i=1,2)$ such that

$$
\begin{aligned}
& m_{i} \leqslant \liminf _{t \rightarrow \infty} N_{i}(t) \leqslant \limsup _{t \rightarrow \infty} N_{i}(t) \leqslant M_{i}, \\
& q_{i} \leqslant \liminf _{t \rightarrow \infty} u_{i}(t) \leqslant \limsup _{t \rightarrow \infty} u_{i}(t) \leqslant Q_{i} .
\end{aligned}
$$

Upper bounds of prey, predator and feedback control population

Proposition 1 Every solution $\left(N_{1}(t), N_{2}(t), u_{1}(t)\right.$, $\left.u_{2}(t)\right)^{\mathrm{T}}$ of system (3) satisfies

$$
\limsup _{t \rightarrow \infty} N_{i}(t) \leqslant N_{i}^{*}, \quad \limsup _{t \rightarrow \infty} u_{i}(t) \leqslant u_{i}^{*},
$$

where

$$
\begin{gathered}
N_{1}^{*}=\frac{b^{M}-a^{l}}{a^{l}}, \quad N_{2}^{*}=\frac{g^{M}-\frac{f^{l}}{\exp \left\{N_{1}^{*}\right\}}}{\frac{f^{l}}{\exp \left\{N_{1}^{*}\right\}}}, \\
u_{i}^{*}=\frac{\alpha_{i}^{M}+\gamma_{i}^{M} \exp \left\{N_{i}^{*}\right\}}{\beta_{i}^{l}}, \quad i=1,2 .
\end{gathered}
$$

Proof: Let $\left(N_{1}(t), N_{2}(t), u_{1}(t), u_{2}(t)\right)^{\mathrm{T}}$ be any solution of system (3), it follows from the first equation of system (3) and the Bernoulli inequality, $\exp \{x\} \geqslant$ $1+x$ for $x \in \mathbb{R}$, we obtain

$$
\begin{aligned}
N_{1}^{\Delta}(t) & \leqslant b(t)-a(t) \exp \left\{N_{1}(t)\right\} \\
& \leqslant b(t)-a(t)\left(N_{1}(t)+1\right) \\
& \leqslant b^{M}-a^{l}-a^{l} N_{1}(t), \quad t \in \mathbb{T} .
\end{aligned}
$$

It follows from Lemma 2 that

$$
\limsup _{t \rightarrow \infty} N_{1}(t) \leqslant \frac{b^{M}-a^{l}}{a^{l}}:=N_{1}^{*} .
$$

Now, for any $\epsilon>0$, there exists a $t_{0} \in \mathbb{T}$ such that $N_{1}(t) \leqslant N_{1}^{*}+\epsilon$ for all $t \geqslant t_{0}$. Then, from the second equation of system (3), we obtain

$$
\begin{aligned}
N_{2}^{\Delta}(t) & \leqslant g(t)-f(t) \exp \left\{N_{2}(t)-N_{1}(t)\right\} \\
& \leqslant g(t)-\frac{f(t)}{\exp \left\{N_{1}^{*}\right\}}\left(N_{2}(t)+1\right) \\
& \leqslant g^{M}-\frac{f^{l}}{\exp \left\{N_{1}^{*}\right\}}-\frac{f^{l}}{\exp \left\{N_{1}^{*}\right\}} N_{2}(t), \quad t \in \mathbb{T} .
\end{aligned}
$$

It follows from Lemma 2 that

$$
\limsup _{t \rightarrow \infty} N_{2}(t) \leqslant \frac{g^{M}-\frac{f^{l}}{\exp \left\{N_{1}^{*}\right\}}}{\frac{f^{l}}{\exp \left\{N_{1}^{*}\right\}}}:=N_{2}^{*} .
$$

For any $\epsilon>0$ and there exists a $t_{1}>t_{0}$ in the above inequality, which leads to

$$
N_{2}(t) \leqslant N_{2}^{*}+\epsilon, \quad t>t_{1}, t \in \mathbb{T} .
$$

Similarly, from the third equation of system (3), we have for $t>t_{1}, t \in \mathbb{T}, i=1,2$

$$
\begin{aligned}
u_{i}^{\Delta}(t) & \leqslant \alpha_{i}(t)-\beta_{i}(t) u_{i}(t)+\gamma_{i}(t) \exp \left\{N_{i}^{*}+\epsilon\right\} \\
& \leqslant \alpha_{i}^{M}-\beta_{i}^{l} u_{i}(t)+\gamma_{i}^{M} \exp \left\{N_{i}^{*}+\epsilon\right\} .
\end{aligned}
$$

Let $\epsilon \rightarrow 0$ in the above inequality, it follows from Lemma 2 that

$$
\limsup _{t \rightarrow \infty} u_{i}(t) \leqslant \frac{\alpha_{i}^{M}+\gamma_{i}^{M} \exp \left\{N_{i}^{*}\right\}}{\beta_{i}^{l}}:=u_{i}^{*}, \quad i=1,2 .
$$

The proof is completed. 


\section{Lower bounds of prey, predator and feedback control population}

Proposition 2 Assume that $\left(H_{1}\right) \quad b^{l}>d^{M} u_{1}^{*}$ and $\left(H_{2}\right) g^{l}>p^{M} u_{2}^{*}$, then every solution $\left(N_{1}(t), N_{2}(t), u_{1}(t), u_{2}(t)\right)^{\mathrm{T}}$ of system (3) satisfies

$$
\liminf _{t \rightarrow \infty} N_{i}(t) \geqslant N_{i *}, \quad \liminf _{t \rightarrow \infty} u_{i}(t) \geqslant u_{i *}
$$

where

$$
\begin{gathered}
N_{1 *}=\ln \frac{b^{l}-d^{M} u_{1}^{*}}{a^{M}+\frac{c^{M}}{\left(h^{l}\right)^{2} \exp \left\{N_{2}^{*}\right\}}}, \quad N_{2 *}=\ln \frac{g^{l}-p^{M} u_{2}^{*}}{\frac{f^{M}}{\exp \left\{N_{1 *}\right\}}}, \\
u_{i *}=\frac{\alpha_{i}^{l}+\gamma_{i}^{l} \exp \left\{N_{i *}\right\}}{\beta_{i}^{M}}, \quad i=1,2 .
\end{gathered}
$$

Proof: For any $\epsilon>0$, according to Proposition 1, there exists a $t_{2} \in \mathbb{T}$ such that $N_{i}(t) \leqslant N_{i}^{*}+\epsilon, u_{i}(t) \leqslant$ $u_{i}^{*}+\epsilon$ for all $t>t_{2}, i=1,2$. Then for $t>t_{2}$, from the first equation of system (3), we obtain

$$
\begin{aligned}
& N_{1}^{\Delta}(t) \geqslant b(t)-a(t) \exp \left\{N_{1}(t)\right\}-d(t)\left(u_{1}^{*}+\epsilon\right) \\
&-\frac{c(t) \exp \left\{N_{1}(t)\right\} \exp \left\{N_{2}^{*}+\epsilon\right\}}{h(t)^{2} \exp \left\{2\left(N_{2}^{*}+\epsilon\right)\right\}+\exp \left\{2 N_{1}(t)\right\}} \\
& \geqslant b(t)-a(t) \exp \left\{N_{1}(t)\right\}-d(t)\left(u_{1}^{*}+\epsilon\right) \\
&-\frac{c(t) \exp \left\{N_{1}(t)\right\}}{h(t)^{2} \exp \left\{N_{2}^{*}+\epsilon\right\}} \\
& \geqslant b^{l}-d^{M}\left(u_{1}^{*}+\epsilon\right)-\left(a^{M}+\frac{c^{M}}{h^{l 2} \exp \left\{N_{2}^{*}+\epsilon\right\}}\right) \exp \left\{N_{1}(t)\right\} .
\end{aligned}
$$

We claim that for $t>t_{2}$

$$
b^{l}-d^{M}\left(u_{1}^{*}+\epsilon\right)-\left(a^{M}+\frac{c^{M}}{h^{l 2} \exp \left\{N_{2}^{*}+\epsilon\right\}}\right) \exp \left\{N_{1}(t)\right\} \leqslant 0 .
$$

Otherwise, assume that there exists $\tilde{t} \geqslant t_{2}$ such that $b^{l}-d^{M}\left(u_{1}^{*}+\epsilon\right)-\left(a^{M}+\frac{c^{M}}{h^{l 2} \exp \left\{N_{2}^{*}+\epsilon\right\}}\right) \exp \left\{N_{1}(\tilde{t})\right\}>0$ and for any $t \in\left[t_{2}, \tilde{t}\right)_{\mathbb{T}}$,

$b^{l}-d^{M}\left(u_{1}^{*}+\epsilon\right)-\left(a^{M}+\frac{c^{M}}{h^{l 2} \exp \left\{N_{2}^{*}+\epsilon\right\}}\right) \exp \left\{N_{1}(t)\right\} \leqslant 0$.

Hence,

$$
N_{1}(\tilde{t})<\ln \frac{b^{l}-d^{M}\left(u_{1}^{*}+\epsilon\right)}{a^{M}+\frac{c^{M}}{h^{l 2} \exp \left\{N_{2}^{*}+\epsilon\right\}}},
$$

and for any $t \in\left[t_{2}, \tilde{t}\right)_{\mathbb{T}}$

$$
N_{1}(t) \geqslant \ln \frac{b^{l}-d^{M}\left(u_{1}^{*}+\epsilon\right)}{a^{M}+\frac{c^{M}}{h^{l 2} \exp \left\{N_{2}^{*}+\epsilon\right\}}},
$$

which implies $N_{1}^{\Delta}(\tilde{t})<0$. It is a contradiction. Therefore, (4) holds for $t \geqslant t_{2}$. Consequently, for $t \geqslant t_{2}$

$$
N_{1}(t) \geqslant \ln \frac{b^{l}-d^{M}\left(u_{1}^{*}+\epsilon\right)}{a^{M}+\frac{c^{M}}{h^{l 2} \exp \left\{N_{2}^{*}+\epsilon\right\}}} .
$$

Let $\epsilon \rightarrow 0$, then

$$
\liminf _{t \rightarrow \infty} N_{1}(t) \geqslant \ln \frac{b^{l}-d^{M} u_{1}^{*}}{a^{M}+\frac{c^{M}}{h^{2} \exp \left\{N_{2}^{*}\right\}}}:=N_{1 *} .
$$

Now, for any small enough $\epsilon>0$, there exists a $t_{3}>$ $t_{2}$ such that $N_{1}(t) \geqslant N_{1 *}-\epsilon$ and $u_{i}(t) \leqslant u_{1}^{*}+\epsilon$ for all $t \geqslant t_{3}, i=1,2$. Similarly, from the second equation of system (3), we obtain for $t \geqslant t_{3}$

$$
\begin{aligned}
N_{2}^{\Delta}(t) & \geqslant g(t)-f(t) \frac{\exp \left\{N_{2}(t)\right\}}{\exp \left\{N_{1}(t)\right\}}-p(t)\left(u_{2}^{*}+\epsilon\right) \\
& \geqslant g^{l}-p^{M}\left(u_{2}^{*}+\epsilon\right)-\frac{f^{M}}{\exp \left\{N_{1 *}-\epsilon\right\}} \exp \left\{N_{2}(t)\right\} .
\end{aligned}
$$

We claim that for $t>t_{3}$

$$
g^{l}-p^{M}\left(u_{2}^{*}+\epsilon\right)-\frac{f^{M}}{\exp \left\{N_{1 *}-\epsilon\right\}} \exp \left\{N_{2}(t)\right\} \leqslant 0 .
$$

Otherwise, assume that there exists $\tilde{t} \geqslant t_{3}$ such that

$$
g^{l}-p^{M}\left(u_{2}^{*}+\epsilon\right)-\frac{f^{M}}{\exp \left\{N_{1 *}-\epsilon\right\}} \exp \left\{N_{2}(\tilde{t})\right\}>0
$$

and for any $t \in\left[t_{3}, \tilde{t}\right)_{\mathbb{T}}$,

$$
g^{l}-p^{M}\left(u_{2}^{*}+\epsilon\right)-\frac{f^{M}}{\exp \left\{N_{1 *}-\epsilon\right\}} \exp \left\{N_{2}(t)\right\} \leqslant 0 .
$$

Hence,

$$
N_{2}(\tilde{t})<\ln \frac{g^{l}-p^{M}\left(u_{2}^{*}+\epsilon\right)}{\frac{f^{M}}{\exp \left\{N_{1 *}-\epsilon\right\}}},
$$

and for any $t \in\left[t_{3}, \tilde{t}\right)_{\mathbb{T}}$,

$$
N_{2}(t) \geqslant \ln \frac{g^{l}-p^{M}\left(u_{2}^{*}+\epsilon\right)}{\frac{f^{M}}{\exp \left\{N_{1 *}-\epsilon\right\}}},
$$

which implies $N_{2}^{\Delta}(\tilde{t})<0$. It is a contradiction. Therefore, (5) holds for $t \geqslant t_{3}$. Consequently, for $t \geqslant t_{3}$,

$$
N_{2}(t) \geqslant \ln \frac{g^{l}-p^{M}\left(u_{2}^{*}+\epsilon\right)}{\frac{f^{M}}{\exp \left\{N_{1_{*}}-\epsilon\right\}}} .
$$

Let $\epsilon \rightarrow 0$, then

$$
\liminf _{t \rightarrow \infty} N_{2}(t) \geqslant \ln \frac{g^{l}-p^{M} u_{2}^{*}}{\frac{f^{M}}{\exp \left\{N_{1 *}\right\}}}:=N_{2 *} .
$$


Now, for any small enough $\epsilon>0$, there exists a $t_{4} \in$ $\mathbb{T}$ such that $N_{i}(t) \geqslant N_{i *}-\epsilon$ for all $t \geqslant t_{4}, i=1,2$. From the third equation of system (3), we have

$$
\begin{aligned}
u_{i}^{\Delta}(t) & \geqslant a_{i}(t)-\beta_{i}(t) u_{i}(t)+\gamma_{i}(t) \exp \left\{N_{i *}+\epsilon\right\} \\
& \geqslant \alpha_{i}^{l}-\beta_{i}^{M} u_{i}(t)+\gamma_{i}^{l} \exp \left\{N_{i *}-\epsilon\right\}, \quad i=1,2 .
\end{aligned}
$$

It follows from Lemma 2 that

$$
\lim _{t \rightarrow \infty} \inf u_{i}(t) \geqslant \frac{\alpha_{i}^{l}+\gamma_{i}^{l} \exp \left\{N_{i *}-\epsilon\right\}}{\beta_{i}^{M}}, \quad i=1,2 .
$$

Let $\epsilon \rightarrow 0$, we get

$$
\lim _{t \rightarrow \infty} \inf u_{i}(t) \geqslant \frac{\alpha_{i}^{l}+\gamma_{i}^{l} \exp \left\{N_{i *}\right\}}{\beta_{i}^{M}}:=u_{i *}, \quad i=1,2 .
$$

The proof is completed.

\section{Permanence result}

Now the main result of this section is obtained as follows.

Theorem 1 Assume that $\left(H_{1}\right)$ and $\left(H_{2}\right)$ hold, then system (3) is permanent.

Remark 1 If $\mathbb{T}=\mathbb{R}$ or $\mathbb{T}=\mathbb{Z}$, Theorem 1 gives a decision theorem for permanence of continuous or discrete Leslie predator-prey model with feedback controls, respectively. These results were obtained in $[11,15]$. Therefore, our work in this paper extends the corresponding results in $[11,15]$.

\section{UNIFORM ASYMPTOTICAL STABILITY}

Set $\mathbb{T}_{1}=\{t \in \mathbb{T}: \sigma(t)>t\}, \mu^{-}=\inf _{t \in \mathbb{T}_{1}} \mu(t)=$ $\inf _{t \in \mathbb{T}_{1}}[\sigma(t)-t]$ and $\mu^{+}=\sup _{t \in \mathbb{T}_{1}} \mu(t)=$ $\sup _{t \in \mathbb{T}_{1}}[\sigma(t)-t]$.

Theorem 2 Assume that $\left(H_{1}\right)$ and $\left(H_{2}\right)$ hold and suppose further that one of the following cases holds:

$\left(H_{3}\right) \mathbb{T}_{1}=\varnothing$ and $\Xi_{1}>0$, where

$$
\begin{aligned}
\Xi_{1}= & \min \left\{a^{l} \mathrm{e}^{N_{1 *}}-c^{M} H-f^{M} \mathrm{e}^{N_{2}^{*}-N_{1 *}}-\gamma_{1}^{M} \mathrm{e}^{N_{1}^{*},}\right. \\
& \left.f^{l} \mathrm{e}^{N_{2 *}-N_{1}^{*}}-c^{M} H-\gamma_{2}^{M} \mathrm{e}^{N_{2}^{*}}, \beta_{1}^{l}-d^{M}, \beta_{2}^{l}-p^{M}\right\}, \\
H= & \frac{\mathrm{e}^{N_{1}^{*}+N_{2}^{*}}}{\left[\left(h^{l}\right)^{2} \mathrm{e}^{2 N_{2 *}}+\mathrm{e}^{2 N_{1 *}}\right]^{2}} \times \\
& \max \left\{\left|\mathrm{e}^{2 N_{1}^{*}}-\left(h^{l}\right)^{2} \mathrm{e}^{2 N_{2 *}}\right|,\left|\left(h^{M}\right)^{2} \mathrm{e}^{2 N_{2}^{*}}-\mathrm{e}^{2 N_{1 *}}\right|\right\} .
\end{aligned}
$$

$\left(H_{4}\right) \mathbb{T}_{1}=\mathbb{T}$ and $\Xi_{2}>0$, where

$$
\begin{aligned}
\Xi_{2}= & \min \left\{\frac{1}{\mu^{+}}-a^{*}-c^{M} H-f^{M} \mathrm{e}^{N_{2}^{*}-N_{1 *}}-\gamma_{1}^{M} \mathrm{e}^{N_{1}^{*},}\right. \\
& \frac{1}{\mu^{+}}-f^{*}-c^{M} H-\gamma_{2}^{M} \mathrm{e}^{N_{2}^{*}}, \frac{1}{\mu^{+}}-\beta_{1}^{*}-d^{M}, \\
& \left.\frac{1}{\mu^{+}}-\beta_{2}^{*}-p^{M}\right\},
\end{aligned}
$$

$$
\begin{aligned}
& a^{*}=\max \left\{\left|\frac{1}{\mu^{-}}-a^{l} \mathrm{e}^{N_{1 *}}\right|,\left|a^{M} \mathrm{e}^{N_{1}^{*}}-\frac{1}{\mu^{+}}\right|\right\}, \\
& f^{*}=\max \left\{\left|\frac{1}{\mu^{-}}-f^{l} \mathrm{e}^{N_{2 *}-N_{1}^{*}}\right|,\left|f^{M} \mathrm{e}^{N_{2}^{*}-N_{1 *}}-\frac{1}{\mu^{+}}\right|\right\}, \\
& \beta_{i}^{*}=\max \left\{\left|\frac{1}{\mu^{-}}-\beta_{i}^{l}\right|,\left|\beta_{i}^{M}-\frac{1}{\mu^{+}}\right|\right\}, \quad i=1,2 .
\end{aligned}
$$

$\left(H_{5}\right) \mathbb{T}_{1} \neq \varnothing, \mathbb{T}_{1} \neq \mathbb{T}, \Xi_{1}>0$ and $\Xi_{2}>0$,

then system (3) is uniformly asymptotically stable.

Proof: Suppose that $X(t)=\left(N_{1}(t), N_{2}(t), u_{1}(t)\right.$, $\left.u_{2}(t)\right)^{\mathrm{T}}$ and $\bar{X}(t)=\left(\bar{N}_{1}(t), \bar{N}_{2}(t), \bar{u}_{1}(t), \bar{u}_{2}(t)\right)^{\mathrm{T}}$ are any two solutions of system (3), it has

$$
\begin{aligned}
N_{1}^{\Delta}(t) & =b(t)-a(t) \exp \left\{N_{1}(t)\right\}-d(t) u_{1}(t) \\
& -\frac{c(t) \exp \left\{N_{1}(t)+N_{2}(t)\right\}}{h^{2}(t) \exp \left\{2 N_{2}(t)\right\}+\exp \left\{2 N_{1}(t)\right\}}, \\
N_{2}^{\Delta}(t) & =g(t)-f(t) \exp \left\{N_{2}(t)-N_{1}(t)\right\}-p(t) u_{2}(t), \\
u_{i}^{\Delta}(t) & =\alpha_{i}(t)-\beta_{i}(t) u_{i}(t)+\gamma_{i}(t) \exp \left\{N_{i}(t)\right\}, \\
\bar{N}_{1}^{\Delta}(t) & =b(t)-a(t) \exp \left\{\bar{N}_{1}(t)\right\} \\
& -\frac{c(t) \exp \left\{\bar{N}_{1}(t)+\bar{N}_{2}(t)\right\}}{h^{2}(t) \exp \left\{2 \bar{N}_{2}(t)\right\}+\exp \left\{2 \bar{N}_{1}(t)\right\}}-d(t) \bar{u}_{1}(t), \\
\bar{N}_{2}^{\Delta}(t) & =g(t)-f(t) \exp \left\{\bar{N}_{2}(t)-\bar{N}_{1}(t)\right\}-p(t) \bar{u}_{2}(t), \\
\bar{u}_{i}^{\Delta}(t) & =\alpha_{i}(t)-\beta_{i}(t) \bar{u}_{i}(t)+\gamma_{i}(t) \exp \left\{\bar{N}_{i}(t)\right\}, i=1,2 .
\end{aligned}
$$

Considering the Lyapunov function $V(t, X, \bar{X})$ on $\mathbb{T}$ defined by

$$
V(t)=V(t, X, \bar{X})=\sum_{i=1}^{2}\left|N_{i}(t)-\bar{N}_{i}(t)\right|+\sum_{i=1}^{2}\left|u_{i}(t)-\bar{u}_{i}(t)\right| .
$$

Case 1: $\mathbb{T}_{1}=\varnothing$, i.e., $\sigma(t)=t, \forall t \in \mathbb{T}$. In view of system (6), we get

$$
\begin{aligned}
\left(N_{1}(t)-\bar{N}_{1}(t)\right)^{\prime}= & -a(t)\left(\exp \left\{N_{1}(t)\right\}-\exp \left\{\bar{N}_{1}(t)\right\}\right) \\
& -d(t)\left(u_{1}(t)-\bar{u}_{1}(t)\right) \\
& -c(t)\left[\frac{\exp \left\{N_{1}(t)+N_{2}(t)\right\}}{h^{2}(t) \exp \left\{2 N_{2}(t)\right\}+\exp \left\{2 N_{1}(t)\right\}}\right. \\
& \left.-\frac{\exp \left\{\bar{N}_{1}(t)+\bar{N}_{2}(t)\right\}}{h^{2}(t) \exp \left\{2 \bar{N}_{2}(t)\right\}+\exp \left\{2 \bar{N}_{1}(t)\right\}}\right], \\
\left(N_{2}(t)-\bar{N}_{2}(t)\right)^{\prime}= & -f(t)\left(\exp \left\{N_{2}(t)-N_{1}(t)\right\}\right. \\
& \left.-\exp \left\{\bar{N}_{2}(t)-\bar{N}_{1}(t)\right\}\right)-p(t)\left(u_{2}(t)-\bar{u}_{2}(t)\right), \\
\left(u_{i}(t)-\bar{u}_{i}(t)\right)^{\prime}= & -\beta_{i}(t)\left(u_{i}(t)-\bar{u}_{i}(t)\right) \\
& +\gamma_{i}(t)\left(\exp \left\{N_{i}(t)\right\}-\exp \left\{\bar{N}_{i}(t)\right\}\right), \quad i=1,2 .
\end{aligned}
$$

Calculating the right derivative $D^{+} V^{\Delta}$ of $V$ along the solution of system (7) leads to

$$
D^{+} V^{\Delta}(t)=V_{1}(t)+V_{2}(t)+V_{3}(t)+V_{4}(t),
$$

where

$$
\begin{array}{ll}
V_{1}(t)=D^{+}\left|N_{1}(t)-\bar{N}_{1}(t)\right|, & V_{2}(t)=D^{+}\left|N_{2}(t)-\bar{N}_{2}(t)\right|, \\
V_{3}(t)=D^{+}\left|u_{1}(t)-\bar{u}_{1}(t)\right|, & V_{4}(t)=D^{+}\left|u_{2}(t)-\bar{u}_{2}(t)\right| .
\end{array}
$$


By using the mean value theorem, it yields from system (7) that

$$
\begin{aligned}
V_{1}(t)= & \operatorname{sgn}\left(N_{1}(t)-\bar{N}_{1}(t)\right)\left(N_{1}(t)-\bar{N}_{1}(t)\right)^{\prime} \\
\leqslant & -\left[a^{l} \mathrm{e}^{N_{1 *}}-c^{M} H\right]\left|N_{1}(t)-\bar{N}_{1}(t)\right| \\
& +c^{M} H\left|N_{2}(t)-\bar{N}_{2}(t)\right|+d^{M}\left|u_{1}(t)-\bar{u}_{1}(t)\right|, \\
V_{2}(t)= & \operatorname{sgn}\left(N_{2}(t)-\bar{N}_{2}(t)\right)\left(N_{2}(t)-\bar{N}_{2}(t)\right)^{\prime} \\
\leqslant & -f^{l} \mathrm{e}^{N_{2 *}-N_{1}^{*}}\left|N_{2}(t)-\bar{N}_{2}(t)\right| \\
+ & f^{M} \mathrm{e}^{N_{2}^{*}-N_{1 *}}\left|N_{1}(t)-\bar{N}_{1}(t)\right|+p^{M}\left|u_{2}(t)-\bar{u}_{2}(t)\right|,
\end{aligned}
$$

and, for $i=1,2$,

$$
\begin{aligned}
V_{i}(t) & =\operatorname{sgn}\left(u_{i}(t)-\bar{u}_{i}(t)\right)\left(u_{i}(t)-\bar{u}_{i}(t)\right)^{\prime} \\
& \leqslant-\beta_{i}^{l}\left|u_{i}(t)-\bar{u}_{i}(t)\right|+\gamma_{i}^{M} \mathrm{e}^{N_{i}^{*}}\left|N_{i}(t)-\bar{N}_{i}(t)\right| .
\end{aligned}
$$

Therefore, for $t \in \mathbb{T} \backslash \mathbb{T}_{1}$,

$$
\begin{aligned}
& D^{+} V^{\Delta}(t)=V_{1}(t)+V_{2}(t)+V_{3}(t)+V_{4}(t) \\
& \quad \leqslant-\left[a^{l} \mathrm{e}^{N_{1 *}}-c^{M} H-f^{M} \mathrm{e}^{N_{2}^{*}-N_{1 *}}-\gamma_{1}^{M} \mathrm{e}^{N_{1}^{*}}\right]\left|N_{1}(t)-\bar{N}_{1}(t)\right| \\
& \quad-\left[f^{l} \mathrm{e}^{N_{2 *}-N_{1}^{*}}-c^{M} H-\gamma_{2}^{M} \mathrm{e}^{N_{2}^{*}}\right]\left|N_{2}(t)-\bar{N}_{2}(t)\right| \\
& \quad-\left[\beta_{1}^{l}-d^{M}\right]\left|u_{1}(t)-\bar{u}_{1}(t)\right|-\left[\beta_{2}^{l}-p^{M}\right]\left|u_{2}(t)-\bar{u}_{2}(t)\right| \\
& \quad \leqslant-\Xi_{1} V(t) .
\end{aligned}
$$

Integrating (8) from 0 to $t$ leads to

$$
\begin{array}{r}
V(t)+\Xi_{1} \int_{0}^{t}\left[\sum_{i=1}^{2}\left|N_{i}(s)-\bar{N}_{i}(s)\right|+\sum_{i=1}^{2}\left|u_{i}(s)-\bar{u}_{i}(s)\right|\right] \mathrm{d} s \\
\leqslant V(0)<\infty
\end{array}
$$

for all $t \geqslant 0$, that is,

$$
\int_{0}^{t}\left[\sum_{i=1}^{2}\left|N_{i}(s)-\bar{N}_{i}(s)\right|+\sum_{i=1}^{2}\left|u_{i}(s)-\bar{u}_{i}(s)\right|\right] \mathrm{d} s<\infty,
$$

which implies

$$
\lim _{s \rightarrow \infty}\left[\sum_{i=1}^{2}\left|N_{i}(s)-\bar{N}_{i}(s)\right|+\sum_{i=1}^{2}\left|u_{i}(s)-\bar{u}_{i}(s)\right|\right]=0 .
$$

Thus, system (3) is uniformly asymptotically stable. Case 2: $\mathbb{T}_{1}=\mathbb{T}$, i.e., $\sigma(t)>t, \forall t \in \mathbb{T}$. In view of system (6), we get

$$
\left\{\begin{array}{c}
N_{1}(\sigma(t))-\bar{N}_{1}(\sigma(t))=N_{1}(t)-\bar{N}_{1}(t)-\mu(t) d(t)\left(u_{1}(t)-\bar{u}_{1}(t)\right) \\
-\mu(t) a(t)\left(\exp \left\{N_{1}(t)\right\}-\exp \left\{\bar{N}_{1}(t)\right\}\right) \\
-\mu(t) c(t)\left[\frac{\exp \left\{N_{1}(t)+N_{2}(t)\right\}}{h^{2}(t) \exp \left\{2 N_{2}(t)\right\}+\exp \left\{2 N_{1}(t)\right\}}\right. \\
\left.-\frac{\exp \left\{\bar{N}_{1}(t)+\bar{N}_{2}(t)\right\}}{h^{2}(t) \exp \left\{2 \bar{N}_{2}(t)\right\}+\exp \left\{2 \bar{N}_{1}(t)\right\}}\right], \\
N_{2}(\sigma(t))-\bar{N}_{2}(\sigma(t))=N_{2}(t)-\bar{N}_{2}(t)-\mu(t) p(t)\left(u_{2}(t)-\bar{u}_{2}(t)\right) \\
-\mu(t) f(t)\left(\exp \left\{N_{2}(t)-N_{1}(t)\right\}-\exp \left\{\bar{N}_{2}(t)-\bar{N}_{1}(t)\right\}\right), \\
u_{i}(\sigma(t))-\bar{u}_{i}(\sigma(t))=\left(1-\mu(t) \beta_{i}(t)\right)\left(u_{i}(t)-\bar{u}_{i}(t)\right) \\
+\mu(t) \gamma_{i}(t)\left(\exp \left\{N_{i}(t)\right\}-\exp \left\{\bar{N}_{i}(t)\right\}\right), \quad i=1,2 .
\end{array}\right.
$$

Calculating the right derivative $D^{+} V^{\Delta}$ of $V$ along the solution of system (9) leads to

$$
D^{+} V^{\Delta}(t)=\tilde{V}_{1}(t)+\tilde{V}_{2}(t)+\tilde{V}_{3}(t)+\tilde{V}_{4}(t),
$$

where

$$
\begin{aligned}
\tilde{V}_{1}(t)=\mid & N_{1}(t)-\left.\bar{N}_{1}(t)\right|^{\Delta} \\
= & \frac{\left|N_{1}(\sigma(t))-\bar{N}_{1}(\sigma(t))\right|-\left|N_{1}(t)-\bar{N}_{1}(t)\right|}{\mu(t)}, \\
\tilde{V}_{2}(t)=\mid & \left|N_{2}(t)-\bar{N}_{2}(t)\right|^{\Delta} \\
= & \frac{\left|N_{2}(\sigma(t))-\bar{N}_{2}(\sigma(t))\right|-\left|N_{2}(t)-\bar{N}_{2}(t)\right|}{\mu(t)},
\end{aligned}
$$

$$
\begin{array}{r}
\tilde{V}_{3}(t)=\left|u_{1}(t)-\bar{u}_{1}(t)\right|^{\Delta} \\
=\frac{\left|u_{1}(\sigma(t))-\bar{u}_{1}(\sigma(t))\right|-\left|u_{1}(t)-\bar{u}_{1}(t)\right|}{\mu(t)}, \\
\tilde{V}_{4}(t)=\left|u_{2}(t)-\bar{u}_{2}(t)\right|^{\Delta} \\
=\frac{\left|u_{2}(\sigma(t))-\bar{u}_{2}(\sigma(t))\right|-\left|u_{2}(t)-\bar{u}_{2}(t)\right|}{\mu(t)} .
\end{array}
$$

By the mean value theorem, we have from system (9) that

$$
\begin{aligned}
& \left|N_{1}(\sigma(t))-\bar{N}_{1}(\sigma(t))\right| \leqslant \mu(t)\left(a^{*}+c^{M} H\right)\left|N_{1}(t)-\bar{N}_{1}(t)\right| \\
& +\mu(t) c^{M} H\left|N_{2}(t)-\bar{N}_{2}(t)\right| \\
& +\mu(t) d^{M}\left|u_{1}(t)-\bar{u}_{1}(t)\right| \text {, } \\
& \left|N_{2}(\sigma(t))-\bar{N}_{2}(\sigma(t))\right| \leqslant \mu(t) f^{*}\left|N_{2}(t)-\bar{N}_{2}(t)\right| \\
& +\mu(t) f^{M} \mathrm{e}^{N_{2}^{*}-N_{1 *}}\left|N_{1}(t)-\bar{N}_{1}(t)\right| \\
& +\mu(t) p^{M}\left|u_{2}(t)-\bar{u}_{2}(t)\right|, \\
& +\mu(t) \gamma_{i}^{M} \mathrm{e}^{N_{i}^{*}}\left|N_{i}(t)-\bar{N}_{i}(t)\right|, \quad i=1,2 .
\end{aligned}
$$

By (10)-(12), we gain

$$
\begin{aligned}
& \tilde{V}_{1}(t) \leqslant-\frac{1-\mu(t)\left(a^{*}+C^{M} H\right)}{\mu(t)}\left|N_{1}(t)-\bar{N}_{1}(t)\right| \\
&+c^{M} H\left|N_{2}(t)-\bar{N}_{2}(t)\right|+d^{M}\left|u_{1}(t)-\bar{u}_{1}(t)\right|, \\
& \tilde{V}_{2}(t) \leqslant-\frac{1-\mu(t) f^{*}}{\mu(t)}\left|N_{2}(t)-\bar{N}_{2}(t)\right| \\
& \quad+f^{M} \mathrm{e}^{N_{2}^{*}-N_{1 *}}\left|N_{1}(t)-\bar{N}_{1}(t)\right|+p^{M}\left|u_{2}(t)-\bar{u}_{2}(t)\right|,
\end{aligned}
$$




$$
\begin{aligned}
\tilde{V}_{i}(t) \leqslant-\frac{1-\mu(t) \beta_{i}^{*}}{\mu(t)} & \left|u_{i}(t)-\bar{u}_{i}(t)\right| \\
& +\gamma_{i}^{M} \mathrm{e}^{N_{i}^{*}}\left|N_{i}(t)-\bar{N}_{i}(t)\right|, \quad i=1,2 .
\end{aligned}
$$

Therefore, for $t \in \mathbb{T}_{1}$,

$$
\begin{aligned}
& D^{+} V^{\Delta}(t)=\tilde{V}_{1}(t)+\tilde{V}_{2}(t)+\tilde{V}_{3}(t)+\tilde{V}_{4}(t) \\
& \leqslant- {\left[\frac{1}{\mu^{+}}-a^{*}-c^{M} H-f^{M} \mathrm{e}^{N_{2}^{*}-N_{1 *}}-\gamma_{1}^{M} \mathrm{e}^{N_{1}^{*}}\right]\left|N_{1}(t)-\bar{N}_{1}(t)\right| } \\
&- {\left[\frac{1}{\mu^{+}}-f^{*}-c^{M} H-\gamma_{2}^{M} \mathrm{e}^{N_{2}^{*}}\right]\left|N_{2}(t)-\bar{N}_{2}(t)\right| } \\
&-\left[\frac{1}{\mu^{+}}-\beta_{1}^{*}-d^{M}\right]\left|u_{1}(t)-\bar{u}_{1}(t)\right| \\
& \quad-\left[\frac{1}{\mu^{+}}-\beta_{2}^{*}-p^{M}\right]\left|u_{2}(t)-\bar{u}_{2}(t)\right| \leqslant-\Xi_{2} V(t) . \quad(13)
\end{aligned}
$$

Integrating (13) from 0 to $t$ leads to

$$
\begin{gathered}
V(t)+\Xi_{2} \int_{0}^{t}\left[\sum_{i=1}^{2}\left|N_{i}(s)-\bar{N}_{i}(s)\right|+\sum_{i=1}^{2}\left|u_{i}(s)-\bar{u}_{i}(s)\right|\right] \mathrm{d} s \\
\leqslant V(0)<\infty,
\end{gathered}
$$

that is,

$$
\int_{0}^{t}\left[\sum_{i=1}^{2}\left|N_{i}(s)-\bar{N}_{i}(s)\right|+\sum_{i=1}^{2}\left|u_{i}(s)-\bar{u}_{i}(s)\right|\right] \mathrm{d} s<\infty,
$$

which implies

$$
\lim _{s \rightarrow \infty}\left[\sum_{i=1}^{2}\left|N_{i}(s)-\bar{N}_{i}(s)\right|+\sum_{i=1}^{2}\left|u_{i}(s)-\bar{u}_{i}(s)\right|\right]=0 .
$$

Thus, system (3) is uniformly asymptotically stable. Case 3: $\mathbb{T}_{1} \neq \varnothing, \mathbb{T}_{1} \neq \mathbb{T}$. From (7) and (9), $V(t) \leqslant$ $-\Xi V(t), \forall t \in \mathbb{T}$. Similar to the above argument, we can easily obtain uniform asymptotical stability of system (3). This completes the proof.

Remark 2 In Theorem 2, condition $\left(H_{3}\right)$ corresponds to $\mathbb{T}=\mathbb{R}$, condition $\left(H_{4}\right)$ corresponds to $\mathbb{T}=\mathbb{Z}$ and condition $\left(H_{5}\right)$ corresponds to $\mathbb{T} \neq \mathbb{R}$ and $\mathbb{T} \neq \mathbb{Z}$. When $\mathbb{T}=\mathbb{R}$ or $\mathbb{T}=\mathbb{Z}$, Theorem 2 gives a decision theorem for uniform asymptotical stability of continuous or discrete Leslie predator-prey model with feedback controls, respectively. These results were obtained in $[11,15]$. Therefore, our work in this paper extends the corresponding results in $[11,15]$.

\section{BIOLOGICAL MEANINGS}

Theorems 1 and 2 imply the following biological indications:

(1) The prey and predator populations have the upper bounds if the growth rate of prey exceeds the prey death rate, and the product of the growth rate of predator and the maximum upper bounds of prey exceed the maximum value $f$ which represents per capita reduction rate of prey $N_{1}$.

(2) The prey and predator populations have the lower bounds if the growth rate of prey (i.e., $b$ ) exceeds the feedback control $d u_{1}$ and the growth rate of predator (i.e., $g$ ) exceeds the feedback control $p u_{2}$.

(3) The maximum upper bounds of prey and predator populations are $\left(b^{M}-a^{l}\right) / a^{l}$ and $\left(g^{M}-\frac{f^{l}}{N_{1}^{*}}\right) / \frac{f^{l}}{N_{1}^{*}}$, respectively. It is clear that predator population grows as prey population grows.

(4) From the definitions of $N_{1}^{*}$ and $N_{2}^{*}$, the growth rates of prey and predator, the prey death rate and the maximum value $f$ which represents per capita reduction rate of prey $N_{1}$ could effectively regulate the upper bounds of prey and predator populations, respectively. The upper bounds of prey and predator populations grow as the growth rates of prey and predator grow, and the prey death rate and the maximum value $f$ reduce, respectively.

(5) The minimum lower bounds of prey and predator populations are $\ln \left[\left(b^{l}-d^{M} u_{1}^{*}\right) /\left(a^{M}+\frac{c^{M}}{\left(h^{l}\right)^{2} \exp \left\{N_{2}^{*}\right\}}\right)\right] \quad$ and $\ln \left[\left(g^{l}-p^{M} u_{2}^{*}\right) / \frac{f^{M}}{N_{1 *}}\right]$, respectively. It is easy to observe that the lower bound of prey population grows as the upper bound of predator population grows. Meanwhile, the lower bound of predator population grows as the lower bound of prey population grows.

(6) From conditions $\left(H_{5}\right)-\left(H_{7}\right)$, the uniform asymptotical stability of system (3) is influenced by the coefficients of the system. If the maximum value $c$ of the attainable per capita reduction rate of $N_{1}$, the interference parameter $h$ and the feedback control coefficients (i.e., $d, p, \gamma_{1}, \gamma_{2}$ ) are small enough, then system (3) is uniformly asymptotically stable. 

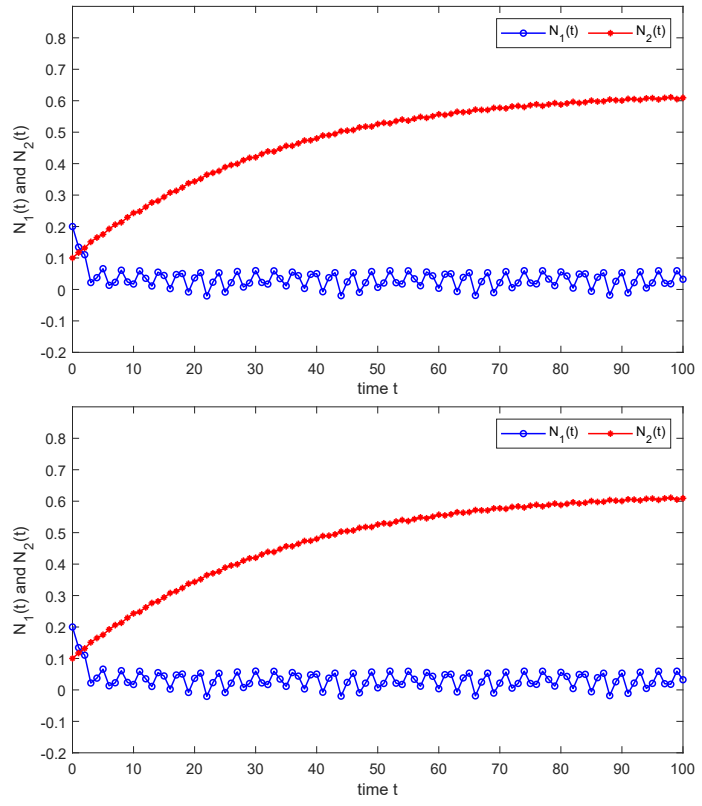

Fig. 1 Permanence of $\left(N_{1}(t), N_{2}(t), u_{1}(t), u_{2}(t)\right)^{\mathrm{T}}$ for system (14).

\section{NUMERICAL ILLUSTRATIONS}

Example 1 Regarding the following Leslie-Gower system with feedback controls:

$$
\left\{\begin{aligned}
& N_{1}^{\Delta}(t)= 4+|\sin t|-4.5 \exp \left\{N_{1}(t)\right\}-0.002 u_{1}(t) \\
&-\frac{0.001 \exp \left\{N_{1}(t)+N_{2}(t)\right\}}{0.01 \exp \left\{2 N_{2}(t)\right\}+\exp \left\{2 N_{1}(t)\right\}} \\
& N_{2}^{\Delta}(t)= 0.3+0.1|\cos t|-0.2 \exp \left\{N_{2}(t)-N_{1}(t)\right\} \\
&-0.001 u_{2}(t), \\
& u_{i}^{\Delta}(t)=-0.2 u_{i}(t)+0.01 \exp \left\{N_{i}(t)\right\} \\
& t \in \mathbb{T}=0.1 \mathbb{Z}=\{0.1 k: k \in \mathbb{Z}\}, i=1,2 .
\end{aligned}\right.
$$

Corresponding to system (3), Theorems 1 and 2, $N_{1}^{*}=0.11, N_{2}^{*}=1.2321, u_{1}^{*}=0.0558, u_{2}^{*}=0.1714$, $N_{1 *}=-0.5130, N_{2 *}=-0.1075, u_{1 *}=0.0299, u_{2 *}=$ 0.0449 . Clearly, $\left(H_{1}\right)-\left(H_{2}\right)$ in Theorem 1 are satisfied. By Theorem 1, system (14) is permanent, which can be seen in Fig. 1.

Further, $a^{*}=7.3058, f^{*}=9.8391, \quad \beta_{1}^{*}=$ $\beta_{2}^{*}=9.8, H=35.2780, \Xi_{2}=\min \{1.5082,0.0913$, $0.1980,0.1990\}=0.0913>0$. So $\left(H_{4}\right)$ in Theorem 2 holds. By Theorem 2, system (14) is uniformly asymptotically stable, as shown in Fig. 2 and Fig. 3.

Acknowledgements: This work is supported by National Nature Science Foundation of China (Nos. 11461082, 61903323 and 11961078), the Natural Scientific Research Fund Project of Yunnan Province
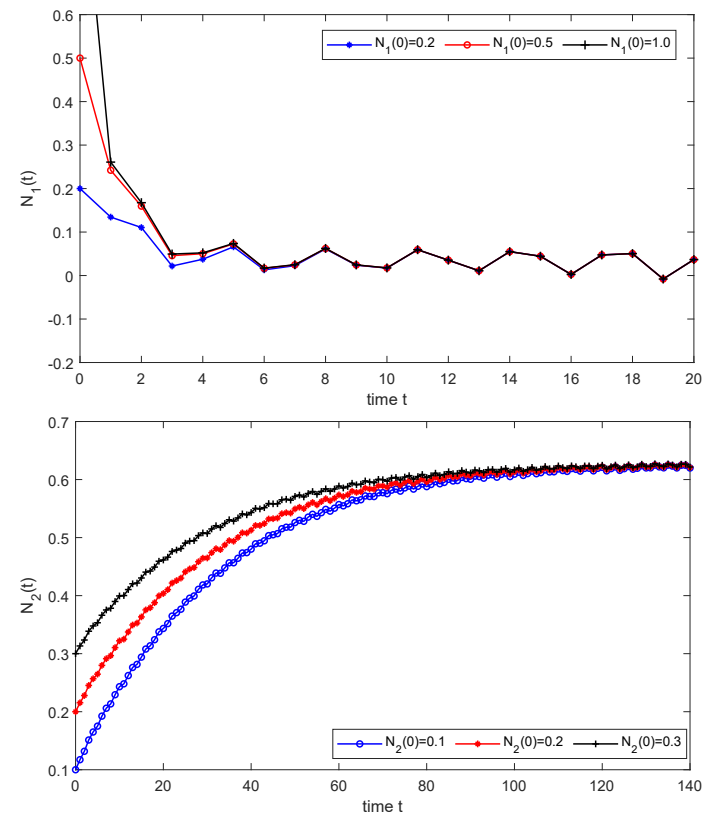

Fig. 2 Uniform asymptotical stability of $N_{1}$ and $N_{2}$ for system (14).
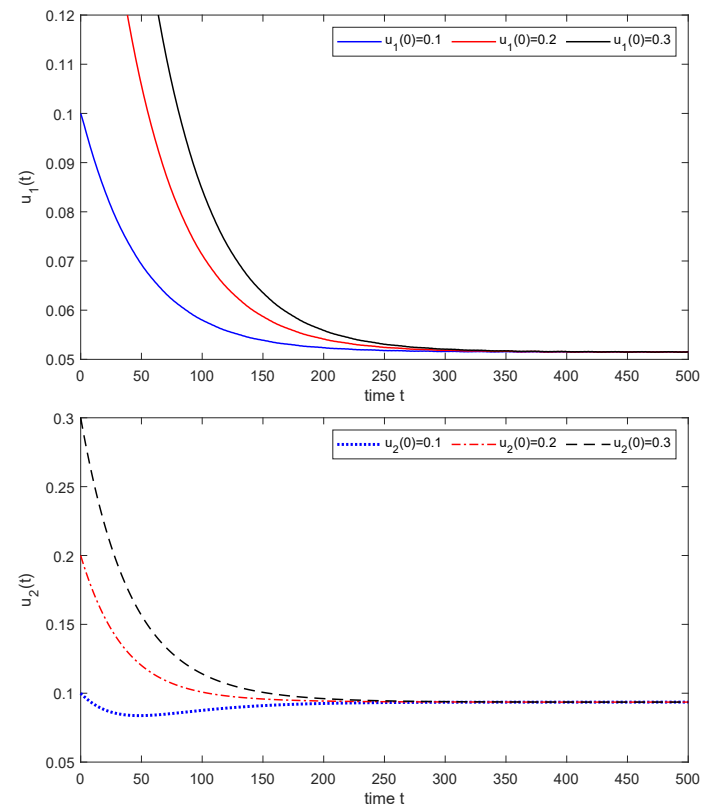

Fig. 3 Uniform asymptotical stability of $u_{1}$ and $u_{2}$ for system (14).

(Grant No. 2018FH001-012) and the Construction Plan of Key Laboratory of Institutions of Higher Education in Yunnan Province. 


\section{REFERENCES}

1. Leslie PH (1948) Some further notes on the use of matrices in population mathematics. Biometrika $\mathbf{3 5}$, 213-245.

2. Zhang F, Li Y, Li C (2019) Hopf bifurcation in a delayed diffusive Leslie-Gower predator-prey model with herd behavior. Int $J$ Bifurcat Chaos 29, ID 1950055.

3. Du Y, Niu B, Wei J (2019) Two delays induce Hopf bifurcation and double Hopf bifurcation in a diffusive Leslie-Gower predator-prey system. Chaos 29, ID 013101.

4. Feng X, Song Y, Liu J, Wang G (2018) Permanence, stability, and coexistence of a diffusive predator-prey model with modified Leslie-Gower and B-D functional response. Adv Differ Equ 2018, ID 314.

5. Liang Z, Zeng X, Pang G, Liang Y (2017) Periodic solution of a Leslie predator-prey system with ratiodependent and state impulsive feedback control. Nonlinear Dyn 89, 2941-2955.

6. Zhang TW, Xiong LL (2020) Periodic motion for impulsive fractional functional differential equations with piecewise Caputo derivative. Appl Math Lett 101, ID 106072.

7. Zhang TW, Yang L, Xu LJ (2020) Stage-structured control on a class of predator-prey system in almost periodic environment. Int J Control 93, 1442-1460.

8. Yu SB, Chen FD (2014) Almost periodic solution of a modified Leslie-Gower predator-prey model with Holling-type II schemes and mutual interference. Int $J$ Biomath 7, ID 1450028.

9. Yue Q (2015) Permanence for a modified LeslieGower predator-prey model with BeddingtonDeAngelis functional response and feedback controls. Adv Differ Equ 2015, ID 81.

10. Chen FD, Cao XH (2008) Existence of almost periodic solution in a ratio-dependent Leslie system with feedback controls. J Math Anal Appl 341, 1399-1412.

11. Wang Q, Zhou J, Wang ZJ, Ding MM, Zhang HY (2011) Existence and attractivity of a periodic solution for a ratio-dependent Leslie system with feed- back controls. Nonlinear Anal RWA 12, 24-33.

12. Rana SMS (2019) Bifurcations and chaos control in a discrete-time predator-prey system of Leslie type. $J$ Appl Anal Comput 9, 31-44.

13. Atabaigi A (2017) Bifurcation and chaos in a discrete time predator-prey system of Leslie type with generalized holling type III functional response. $J$ Appl Anal Comput 7, 411-426.

14. Zhang TW, Gan XR (2013) Existence and permanence of almost periodic solutions for Leslie-Gower predator-prey model with variable delays. Electron $J$ Differ Eq 2013, 1-21.

15. Li ZH (2014) Persistence and almost periodic solutions for a discrete ratio-dependent Leslie system with feedback control. Adv Differ Equ 2014, ID 214.

16. Hilger S (1990) Analysis on measure chains: A unified approach to continuous and discrete calculus. Results Math 18, 18-56.

17. Bohner M, Peterson A (2001) Dynamic Equations on Time Scales, Birkhauser, Boston.

18. Bohner M, Peterson A (2003) Advances in Dynamic Equations on Time Scales, Birkhauser, Boston.

19. Xu CJ, Liao MX, Li PL, Liu ZX (2020) Almost automorphic solutions to cellular neural networks with neutral type delays and leakage delays on time scales. Int J Comput Int Sys 13, 1-11.

20. Alam S, Abbas S, Nieto J (2019) Periodic solutions of a nonautonomous Leslie-Gower predator-prey model with non-linear type prey harvesting on time scales. Differ Equ Dyn Sys 27, 357-367.

21. Bartosiewicz Z, Belikov J, Kotta U, Wyrwas M (2019) State feedback linearization of nonlinear control systems on homogeneous time scales. Nonlinear Anal Hybri 65, 69-85.

22. Lakshmikantham V, Vatsala AS (2002) Hybird systems on time scales. J Comput Appl Math 141, 227-235.

23. Zhang HT, Zhang FD (2014) Permanence of an $N$ species cooperation system with time delays and feedback controls on time scales. J Appl Math Comput $46,17-31$. 